Review paper

\title{
Associating liver partition and portal vein ligation for staged hepatectomy (ALPPS) in colorectal liver metastases: review of the literature
}

\author{
Danilo Coco', Silvana Leanza ${ }^{2}$ \\ 'Department of General Surgery, Ospedali Riuniti Marche Nord, Pesaro, Italy \\ 2Department of General Surgery, Carlo Urbani Hospital, Jesi, Ancona, Italy
}

\begin{abstract}
The liver is considered as one of the most common sites of metastasis and a key determining factor of survival in patients with isolated colorectal liver metastasis (CRLM). For longer survival of patients, surgical resection is the only available option. Especially in CRLM bilobar patients, to achieve RO resection, maintaining an adequate volume of the future liver remnant (FLR) is the main technical challenge to avoid post-hepatectomy liver failure (PHLF). As standard procedures in the treatment of patients with severe metastatic liver disease, techniques such as portal vein embolization/portal vein ligation (PVE/PVL) accompanied by two-stage hepatectomy (TSH) have been introduced. These methods, however, have drawbacks depending on the severity of the disease and the capacity of the patient to expand the liver remnant. Eventually, implementation of the novel ALPPS technique ignited excitement among the community of hepatobiliary surgeons because ALPPS challenged the idea of unrespectability and extended the limit of liver surgery and it was reported that FLR hypertrophy of up to $80 \%$ was induced in a shorter time than PVL or PVE. Nonetheless, ALPPS techniques caused serious concerns due to the associated high morbidity and mortality levels of up to $40 \%$ and $15 \%$ respectively, and PHLF and bile leak are critical morbidity- and mortality-related factors. Carefully establishing the associated risk factors of ALPPS has opened up a new dimension in the field of ALPPS technique for improved surgical outcome by carefully choosing patients. The benefit of ALPPS technique is enhanced when performed for young patients with very borderline remnant volume. Adopting ALPPS technical modifications such as middle hepatic vein preservation, surgical management of the hepatoduodenal ligament, the anterior approach and partial ALPPS may lead to the improvement of ALPPS surgical performance. Research findings to validate the translatability of ALPPS' theoretical advantages into real survival benefits are scarce.
\end{abstract}

Key words: ALPPS, FLR, CRLM, PHLF, bile leak, patient selection.

Address for correspondence:

Dr. Danilo Coco, Department of General Surgery, Ospedali Riuniti Marche Nord, Pesaro, Italy, e-mail: webcostruction@msn.com

\section{Introduction}

Over the past 3 decades, the safe removal of substantial tumour load in the liver has been a major focus of laboratory and clinical research for hepatobiliary surgeons [1]. The key reason for poor postoperative outcomes is an inadequate amount of the future liver remnant (FLR), which contributes to liver failure after hepatectomy [2]. Over the last 3 decades, numerous techniques have been developed to induce compensatory hypertrophy of the FLR, thus increasing the chance of resectability and reducing the risk of postoperative complications [3-5]. The first breakthrough is attributed to Masatoshi Makuuchi, who introduced the concept of portal vein embolization (PVE) of the right portal branch in the 1980s to cause hypertrophy of the left side of the liver, making it possible to safely remove large or multiple tumours, often found in the 
right hemiliver and segment IV [6]. Most followed this procedure rapidly after a series of extensive right-sided hepatectomies to avoid liver failure [2,7]. Today preoperative PVE is considered standard therapy for patients with an inadequate FLR before extended liver resection [8]. Nonetheless, insufficient FLR hypertrophy or progression of the disease after PVE can prevent curative liver resection in up to $20 \%$ of patients $[9,10]$.

Two-stage hepatectomy (TSH) for patients with bilateral multinodular colorectal liver metastases was introduced in the year 2000 [11]. The liver develops in the interval between sequential resection, and the likelihood of postoperative liver failure (PLF) is likely minimized due to the phased approach $[12,13]$. While TSH is well known, failure to proceed to stage 2 is reported to be a problem in up to one-third of patients (8-31\% depending on the series) and occurs due to tumour progression during the regeneration period or inadequate FLR hypertrophy [12].

A novel technique of two-stage liver resection, incorporating portal vein ligation (PVL) and liver transection between the FLR and the deported portion of the liver, has recently been introduced $[4,14]$, which related PVL to the liver partition for staged hepatectomy. Associating liver partition and portal vein ligation for staged hepatectomy (ALPPS) reportedly induces FLR hypertrophy of up to $80 \%$ in a shorter time than PVL or PVE $[14,15]$. This technique, how-
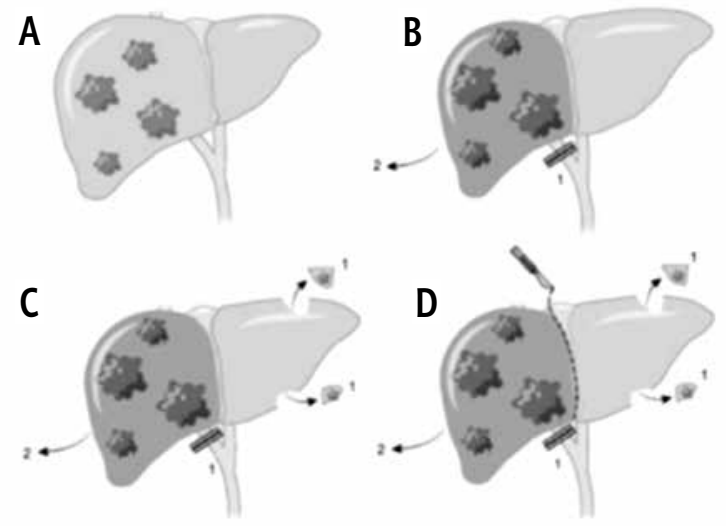

Fig. 1. Visualization of pre- or peri-operative interventions and their effect on liver remnant volume. A) Malignant liver disease; B) embolization/ligation of the right portal branch, (1) resulting in atrophy of the right hemi-liver and compensatory growth of the left hemiliver, which can be removed when appropriate hypertrophy has been achieved (2); C) removal of tumours from the left hemi-liver and occlusion of the right portal branch (1). After 4-6 weeks, the volume of the left hemi-liver is increased and the right hemi-liver can be removed (2); D) removal of tumours from the left hemi-liver, in situ splitting of the hemi-livers, and simultaneous ligation of the right portal vein branch (1). After 1 week, augmented hypertrophy of the left hemi-liver permits removal of the right hemi-liver (2) ever, has caused serious concern due to the associated high morbidity and mortality rates of up to $40 \%$ and $15 \%$ respectively [16]. Despite the benefit of faster tumour-bearing liver resection and higher rates of full resectability compared with traditional TSH, preliminary oncological follow-up results indicated a high rate of recurrence of tumours in patients with ALPPS [17]. So it remains unclear whether accelerated ALPPS regeneration can also induce the proliferation of local and distant micrometastases. Although early results of this novel method have been documented more readily and may provide data on how to improve outcomes, ALPPS's safety compared to traditional FLR hypertrophy approaches, such as PVE and TSH, is still controversial.

In this context, the main objective of this review of the literature is to recapitulate currently available literature information to compare the efficacy of ALPPS against conventional PVE and TSH and to derive strategies to make ALPPS a better surgical option for hepatobiliary surgeons.

\section{Original ALPPS surgical technique and modifications adopted}

ALPPS is a two-stage process. In the first step, the liver is completely mobilized in right lobe dominant disease and the right portal vein branch is ligated. The liver parenchyma is subsequently transected along the falciform ligament. In the first step, wedge resection of any metastatic lesions in the FLR (segments I/II/III) is carried out as well. In order to avoid postoperative adhesions during the waiting period until the next procedure, the right liver lobe and segment IV are then usually wrapped in a plastic bag and the abdomen is closed. The FLR growth is evaluated using $\mathrm{CT}$ volumetry after 7 to 14 days. If the gain of volume appears to be adequate, the subject goes through the second step, usually involving a right trisectionectomy. Reconstruction of the biliary tract, if possible, is done with a hepaticojejunostomy Roux-en-Y [4]. The primary tumour is often resected during the first stage of ALPPS in subjects with synchronous colorectal liver metastases (CRLM) (Fig. 1) [18].

In the literature various ALPPS modifications have been described as more centres of hepatobiliary begin to follow the technique. In ALTPS (associating liver tourniquet and portal vein ligation for staged hepatectomy) proposed by Robles et al. rather than the in-situ liver splitting, a tourniquet was placed along the future line of resection to reduce the flow of blood between the lobes [19]. ALTPS was planned to simplify the first step with a reduced adhesions rate and complications. 
The "anterior method" was just another change recommended to reduce peri-hepatic inflammation after the first stage and decrease the risk of malignant hematologic spread hypothetically [20]. Complete liver mobilization was prevented in the "anterior method" and the hepatoduodenal ligament was kept intact. Complete mobilization of the liver and dissection of all collateral flows assures adequate operating exposure of field with an improved future liver remnant hypertrophy rate, but studies from the literature suggest minimal hepatoduodenal ligament dissection to lessen segment 4 ischemia and biliary leakage risk [21, 22].

Many other technical ALPPS modifications have been named according to the liver segment(s) that will form the future liver remnant $[23,24]$. Only one segment includes FLR in mono-segmental ALPPS $[25,26]$. "Rescue ALPPS" relates to the procedure of ALPPS carried out in subjects who have not achieved an adequate FLR after PVE to undergo TSH in the second step [27-30]. In these subjects, ALPPS is regarded as a last option to facilitate liver hypertrophy. More recently, "laparoscopic first-stage ALPPS", "robotic ALPPS" and "totally laparoscopic ALPPS" are minimally invasive measures which have already been noted to decrease postoperative adhesion rates and overall procedural complications [31-36].

Developments of preoperative liver hypertrophy induction methods and successful therapies of chemotherapy, together with the parenchymal-preserving liver surgery advent, has widened the limits of resectability in CRLM subjects who were originally considered not appropriate for surgical resection due to an unsatisfactory future liver remnant [37]. Moreover, the procedure of ALPPS has raised the bar even further and is a treatment option suggested by the first international expert meeting panel of ALPPS for subjects with colorectal liver metastasis [38].

\section{FLR hypertrophy}

Indeed, ALPPS might be supreme in causing future liver remnant hypertrophy over PVL/PVE in a shorter period of time. In colorectal liver metastasis subjects undergoing ALPPS, liver hypertrophy was noted to be as high as $110.3 \%$ in seven to fourteen days, compared with $20 \%$ to $46 \%$ in two to eight weeks after PVE $[39,40]$. In a Moris et al. meta-analysis, while post-operative FLR, the magnitude of the increase in future liver remnant and post-operative future liver remnant were all comparable between the 2 classes, the rate of kinetic growth was faster with ALPPS against portal vein embolization [41]. The technological variations between the 2 methods and the related underlying physiolog- ical impacts on hepatocytes were suggested as possible reason for the hypertrophy differences. In the first phase, dividing the liver discourages collateral vessels from flowing between the 2 liver sides and retains the shear stress of the portal flow on hepatocytes at its maximum extent, a renowned physiological factor that contributes to regeneration of the liver $[39,42]$. In addition, traumatizing the liver parenchyma in the first phase of ALPPS may increase the mediators of inflammation that trigger regeneration of hepatocytes [42]. Some researchers have recommended that liver hypertrophy at one week does not ensure functional efficiency and may show oedema instead of true hypertrophy and new proliferation of hepatocytes. Hence, the FLR function should be assessed with approaches beyond the normal biochemical profile of the liver and not just the size $[38,43]$.

\section{Feasibility of completion hepatectomy}

An additional possible benefit of ALPPS compared to conventional TSH is the greater efficacy to complete both the hepatectomy phases. Nearly one third of PVL/PVE subjects preceded by TSH for CRLM were unsuccessful to finish second phase resection with $65-75 \%$ feasibility [12]. In contrast, patients' meta-analysis with different underlying liver diseases suffering from ALPPS indicated a success of $97 \%$ feasibility for hepatectomy [14]. In the findings including only colorectal liver metastases subjects this proportion was boosted to as high as $100 \%[22,44,45]$. E.g., $100 \%$ feasibility was noted in a research study conducted by Björnsson et al., although $43 \%$ of subjects faced "rescue ALPPS" [44]. Likewise, in nine recent meta-analysis studies where ALPPS was compared with TSH, the curative-intent resection proportion and possibility of progressing to the second phase in the ALPPS group were higher in comparison to TSH [41].

Progression of tumour during the period between 2 phases and unsatisfactory future liver remnant hypertrophy were confirmed as the 2 main reasons for subject dropout in standard TSH prior to hepatectomy completion [12]. While the shorter duration of time prior to second hepatectomy might enhance overall effectiveness, the shorter interval of time between 2 phases in ALPPS might also hinder the tumour biology assessment, and the detection of micro-metastases is not noticeable in initial imaging studies [46]. This assumption was assisted by a comparatively high tumour recurrence prevalence and a lower disease-free survival (DFS) rate in ALPPS subjects in comparison to conventional TSH $[17,44]$. 


\section{Morbidity and mortality}

The ALPPS method has given rise to serious apprehensions due to increased rates of mortality and morbidity up to $15 \%$ and $40 \%$ correspondingly [16]. Post-hepatectomy liver failure and bile leak were the most significant morbidities correlated with ALPPS. Recognizing the threats allows for a better selection of patients for better results.

Post-hepatectomy liver failure (PHLF) contributed to $75 \%$ mortality associated with ALPPS $[47,48]$. The international registry stated the post-hepatectomy liver failure rate of $9 \%$ using 50-50 criteria [49]. In spite of a rapid increase in median volume of $80 \%$ prior to phase II, $80 \%$ of the subjects with post-hepatectomy liver failure had a future liver remnant of greater than $30 \%$ of the total liver volume prior to phase II. Critics stated that speedy expansion of the remnants in ALPPS was partially due to tissue oedema instead of pure hypertrophy [50]. Also there was a concern whether the volume increase was in parallel with a subsequent increase in function $[51,52]$. Supporting the query were the differences between volume gain and functional evaluation using hepatobiliary scintigraphy. Functional inter-stage increment evaluated by $(99 \mathrm{~m})$ Tc-mebrofenin scan only reached half the volume expansion value [53]. This may explain in part the remarkable rate of post-hepatectomy liver failure after stage II ALPPS regardless of the acceptable volume.

In a registry study of 320 patients to determine factors of risk for 90-day mortality [48], the most important single risk factor was subject age $>60$ years. Inter-stage biochemical parameters also predicted mortality. End-stage liver model disease score $>10$ before stage II and liver failure defined by the International Study Group of Liver Surgery (prolonged international normalized ratio and raised serum bilirubin) were independent risk factors for post-hepatectomy liver failure after ALPPS at day five after stage I [54]. These were simple, objective and reproducible laboratory parameters that permitted clinicians to evaluate the risk of progressing to stage II operation.

Another survey based on registry gathered data developed a risk model for predicting operational mortality after ALPPS [55]. Indicators of stage I poor risk involved biliary malignancy and advanced age. Predictors of stage II comprised severe stage I complication, serum bilirubin level, cumulative stage I risk score and creatinine. Before stage I the selection of subject is perhaps most vital. A risk score of 3 was given to advanced age, whereas a score of 2 was given to biliary tumour and a score of 1 to non-biliary tumour/nonCRLM. A total score of $0,1,2,3,4$ and 5 was related to $3 \%, 5 \%, 9 \%, 15 \%, 24 \%$ and $37 \%$ operative mortality, respectively. An objective mortality prediction was given by the risk model. The message behind it was straightforward: the total score of risk was capped at 2, i.e. $9 \%$ mortality, by avoiding subjects who were old. Moreover, this score led an option to omit or postpone a stage II operation. Serum bilirubin and creatinine level inclusion recommended postponement of stage II to improve liver and renal function and was consistent with higher mortality observation when stage II was followed by a high end-stage liver disease score model [48]. However, it is worth highlighting that adding the stage I cumulative score, i.e., complications of stage I, indication and age, inferred that existence of these poor risk factors regardless of normal renal and liver function still entailed the risk of a stage II operation.

In the early days, bile leakage was one of the commonest reported complications of surgery related to ALPPS. Leakage of bile appeared in $17 \%$ of ALPPS methods as per the registry [47]. The commonest leakage site was at the transection surface of the deported liver owing to segment IV ischemia when the portal vein was ligated, and the parenchymal split was between the parenchymal partition of the left medial and lateral sections. The risk for ALPPS carried out for right trisectionectomy is particularly high [56]. Particularly this was an important issue in right trisectionectomy when segment IV was immediately deprived of both portal and arterial perfusion, which eventually led to necrosis preceded by leakage of bile and septicaemia.

Another factor of risk for leakage of bile is cholangiocarcinoma [57]. Owing to infiltration of the tumour, hilar dissection is difficult technically. Portal lymphadenectomy further deprived the supply of blood from the transection plane [22]. The correlated morbidities of ALPPS were linked closely to the complexity of the procedure. Certainly, independent factors of risk for serious complications from the registry (Clavien-Dindo IIIb or above) were an extended time period for a stage I operation (more than $300 \mathrm{~min}$ ), non-colorectal liver metastases and blood transfusion [47]. ALPPS for hilar cholangiocarcinoma was linked not only with more leakage of bile but also with more operational mortality and post-hepatectomy liver failure $[57,58]$.

\section{Strategies to improve outcomes of ALPPS}

Many novel surgical procedures when first implemented faced unfavourable results. Better results were attained with more careful selection of patient and more sophisticated technical improvements, with increasing experience. A well-recognized international 
registry permitted the systematic collection of information concerning ALPPS [47]. Through better insight and understanding into the treatment method, hepatobiliary surgeons would select better appropriate candidates and further improve their techniques to obtain more favourable results.

\section{Patient selection}

The ALPPS procedure is a great physiological challenge. Though a chronological cut-off may be unrealistic, in subjects with physiological age advancement it is appropriate to skip ALPPS. They have constrained reserves to overcome serious complications. Thus, older subjects are weak ALPPS candidates. Subjects with an age greater than 60 years had higher mortality and showed more severe complications [47] as per the international registry [48].

\section{FLR volume}

A $25 \%$ FLR to estimated standard liver volume (ESLV) ratio is necessary for major hepatectomy to confirm satisfactory postoperative function of the liver in subjects with normal liver [59-61]. The minimum requirement in subjects with underlying liver disease such as cirrhosis, cholestasis, etc. is 30\% [60]. When the future liver remnant is deemed inadequate, TSH with PVE or PVL is a proven technique that promotes FLR hypertrophy of $10 \%$ to $30 \%$ over 4 to 6 weeks [10]. However, unsatisfactory hypertrophy and progression of disease inhibit $10 \%$ to $40 \%$ of subjects from progressing to stage II hepatectomy [16, 62-66].

\section{Disease factor-CRLM}

Colorectal liver metastases is the prominent indication for ALPPS. Over four hundred ALPPS procedures have been conducted worldwide for colorectal liver metastases to date, which includes 220 right trisectionectomies as well as over 180 right hepatectomies [67]. Normal functioning of the liver as well as promising tumour biology are useful for both surgical and oncological results. Registry data confirmed that CRLM is an independent indictor of less serious complications. In $36 \%$ of subjects with colorectal liver metastases major morbidities were observed (Clavien-Dindo 3a or above), which was reduced further to $29 \%$ when only patients with age less than 60 years were chosen [47].

The studies from the literature indicated that chronic liver disease is not an absolute contraindication for ALPPS. Low-grade fibrosis patients are good choices for the treatment and a longer inter-stage interval is de- sired to facilitate adequate hepatic hypertrophy $[68,69]$. A preoperative liver biopsy was proposed by Vivarelli et al. [70] to evaluate the degree of liver fibrosis after PHLF was found in a fibrotic liver subject undergoing ALPPS. The survey of the literature showed that candidates for ALPPS could be successfully selected by analysing surrogate markers representing the degree of liver fibrosis as well as portal hypertension, i.e. platelet count and green clearance of indocyanine. The green retention test for indocyanine was associated with portal hypertension degree $[71,72]$ and mortality in major hepatectomy [73].

\section{Technical improvements}

\section{Preservation of middle hepatic vein}

Parenchymal partition with the middle hepatic vein division was carried out in the initial ALPPS description [74]. With considerable morbidity after ischemic necrosis and leakage of bile, however, it was suggested that the middle hepatic vein could be maintained as the venous outflow of segment IV with no risk to parenchymal hypertrophy [75]. Venous congestion as well as ischemia could be minimized with a patent outflow. Nowadays most hepatobiliary surgeons accept this as their preferred treatment. A questionnaire survey revealed that $70 \%$ of surgeons retained the middle hepatic vein routinely during stage I of ALPPS [76].

\section{Surgical management of hepatoduodenal ligament}

In the classical approach to ALPPS a complete dissection of hilar structures and the hepatoduodenal ligament skeletonization was carried out. This helped in consistent identification of hilar vascular pedicles, but possibly led to the total segment IV devascularisation [22]. Thirty-nine percent of surgeons believed that hepatoduodenal ligament skeletonization was reported in the questionnaire survey [76]. There is presently no consensus regarding the surgical treatment for a hepatoduodenal ligament. In ALPPS, where lymphatic clearance for oncological concerns is not confirmed, importance could be given to restrict dissection of hilar structures in order to prevent possible adverse impacts on segment IV ischemia.

\section{Anterior approach}

The anterior approach to hepatectomy was proposed initially for bulky liver tumours with invasion of surrounding structures [77]. It includes division of the 
portal pedicle and complete parenchymal transection before mobilization of the right liver, reducing bleeding and spillage of tumour at the time of the operation. The anterior approach concept has been implemented to ALPPS $[19,78]$. Without prior mobilization of the right liver, hepatic parenchyma is split up during stage I. The right liver is mobilized in stage II after the right hepatic artery, bile duct and hepatic veins have been divided. The anterior method might be more difficult in the ALPPS setting, because at the time of transection the arterial and biliary pedicles had to be retained. Chan et al. [78] in a prospective series of thirteen subjects found that complete parenchymal splitting with an anterior approach was safe and feasible. In another study carried out by Ardiles et al. it was found that during stage II the frequency of perihepatic adhesions was significantly reduced. $37 \%$ of the registry's ALPPS treatments were conducted using the anterior method [79]. Tumour spillage was negligible with reduced tissue manipulation. Particularly, this was important in an ALPPS setting, where during the inter-stage period the tumour is left in the torso. Before establishment of any oncological advantage of the anterior ALPPS approach further assessment is necessary. With the possible advantages, the anterior method seems to be the favoured treatment, specifically while dealing with a bulky tumour. Moreover, combining a complex method with a more advanced technological approach would be quite difficult. At the time of parenchymal transection more difficult bleeding would be experienced with no reduced vascular control. For hepatobiliary surgeons, anterior approach ALPPS is best reserved for those who do extremely well in conventional hepatectomy in both the anterior approach and ALPPS.

\section{Partial ALPPS}

Based on a canine model, Schlegel et al. [80] inferred that increased regeneration in ALPPS was linked not just to the redistribution of blood flow but also to the existence of secondary circulating factors to tissue damage. After ALPPS, increase in the plasma levels of interleukin (IL)-6 were seen and post-ALPPS plasma was injected into PVL-treated mice, which delivered comparable hypertrophy of the remnants. On the basis of this, Petrowsky et al. [81] recommended an ALPPS technical modification with partial parenchymal partitioning, i.e. $50 \%$ to $80 \%$, in an effort to maintain collateral blood supply and minimise operational morbidity. In stage I, the middle hepatic vein was protected. Termed as partial ALPPS, in the initial series of 6 subjects, the updated technique was correlated with zero mortality and a more desirable complication profile [81]. Partial ALPPS successfully produced the same FLR hypertrophy degree as a complete split (median hypertrophy $60 \%$ vs. $61 \%$ in 7 days). Subsequently, the operative boundary for partial partitioning was described as dissection to the level of the middle hepatic vein as opposed to the inferior vena cava in complete ALPPS [82].

Nevertheless, in chronic hepatitis the efficacy of partial split seemed to be minimal. A comparative study was carried out by Chan et al. [83] for complete as well as partial ALPPS in twenty-five hepatocellular carcinoma (HCC) patients. Partial split was unsuccessful to produce a similar hypertrophy level as that of incomplete split. There was no decrease in perioperative mortality and morbidity. Moreover, the existing proof is based on inadequate experience, and partial ALPPS in larger groups could be further confirmed. Possibly, when functioning of the liver is normal the partial ALPPS is highly successful, and a complete split is difficult technically. If a tumour of larger size is located near the middle hepatic vein or the inferior vena cava, troublesome bleeding from engorged hepatic veins could hinder the parenchymal transmission to the vena cava [83]. Partial ALPPS has decreased risk for bleeding and associated complications. In stage II, the difficult transection is actually better handled when the remnant undergoes hypertrophy and after complete right liver mobilization and division of the arterial and biliary pedicles, the operation is accelerated. Delayed stage II operation and slower hypertrophy are the possible shortcomings.

\section{Summary}

The ALPPS technique has taken many paths since Schnitzbauer et al's original description [4], generating both strong anticipation and scepticism among the surgical community. Where the procedure should fit into the surgeon's weaponry is still not clear. Sandström et al. performed the first randomized controlled multicentre trial comparing traditional TSH and ALPPS in advanced colorectal liver metastases subjects [84]. Subjects suffering from colorectal liver metastases and future liver remnant less than $30 \%$ were appointed randomly to ALPPS ( $n=48$ subjects) and TSH ( $n=49$ subjects). For ALPPS the prime consequence of effective resection of all liver disease against TSH community was higher. In both groups, the incidence of major complications was similar. In the ALPPS group, 5 subjects had to undergo reoperation because of leakage of bile, wound rupture and obstruction in intestine, while only 1 subject needed reoperation in the 
TSH group owing to obstruction in the intestine. Particularly, thirteen subjects in the TSH group dropped out before progressing to second stage hepatectomy. Twelve out of these thirteen subjects underwent rescue ALPPS, while the other 1 subject had progression of the tumour which ruled out a further surgical procedure. After involving these twelve "rescue" ALPPS subjects in the TSH group, the rate of resection in the TSH group was $82 \%$ vs. $92 \%$ in the ALPPS group, although the difference was not statistically significant. 90-day mortality was comparable in the groups of ALPPS and TSH. Given the outcomes of this one small-randomized trial, ALPPS should not be typically regarded as first-line therapy for subjects treated with traditional PVL/PVE procedures to attain a sufficient future liver remnant. Instead, ALPPS must be preserved as an alternative treatment for that patient group which is considered not appropriate for traditional surgical resection.

\section{Conclusions}

In conclusion, ALPPS has been observed to be highly feasible and to significantly promote future liver remnant hypertrophy that extends the patients' eligibility for surgical resection with extensive tumour disorder. In carefully chosen subjects with extensive colorectal liver metastases, ALPPS has superior mortality and morbidity in comparison to TSH. Although traditional strategies including PVL/PVE and TSH should typically be appropriate in most subjects with extensive colorectal liver metastases, ALPPS may play a role in increasing the number of subjects qualified for surgical resection in a group of subjects. In addition, ALPPS questioned the unrespectability concept and extended the scope of hepatic surgery. Mortality is mostly a PHLF outcome and can be lessened with meticulous selection of subjects. The advantage of ALPPS is increased when implemented for young subjects with a very marginal remnant volume. To enhance the effectiveness of ALPPS surgery several technical changes have been recommended. However, studies to confirm the translatability of theoretical advantages into actual survival benefits are scant.

\section{Disclosure}

The authors declare no conflict of interest.

\section{References}

1. de Santibanes E, Clavien PA. Playing Play-Doh to prevent postoperative liver failure: the "ALPPS" approach. Ann Surg 2012; 255: 415-417.
2. Clavien PA, Petrowsky H, DeOliveira ML, et al. Strategies for safer liver surgery and partial liver transplantation. N Engl J Med 2007; 356: 1545-1559.

3. Honjo I, Suzuki T, Ozawa K, et al. Ligation of a branch of the portal vein for carcinoma of the liver. Am J Surg 1975; 130: 296302.

4. Schnitzbauer AA, Lang SA, Goessmann H, et al. Right portal vein ligation combined with in situ splitting induces rapid left lateral liver lobe hypertrophy enabling 2-staged extended right hepatic resection in small for-size settings. Ann Surg 2012; 255: 405-414.

5. Kinoshita H, Sakai K, Hirohashi K, et al. Preoperative portal vein embolization for hepatocellular carcinoma. World J Surg 1986; 10: 803-808.

6. Makuuchi M, Thai BL, Takayasu K, et al. Preoperative portal embolization to increase safety of major hepatectomy for hilar bile duct carcinoma: a preliminary report. Surgery 1990; 107: 521-527.

7. Clavien PA, Oberkofler CE, Raptis DA, et al. What is critical for liver surgery and partial liver transplantation: size or quality? Hepatology 2010; 52: 715-729.

8. Pamecha V, Nedjat-Shokouhi B, GurusamyK, et al. Prospective evaluation of two-stage hepatectomy combined with selective portal vein embolization and systemic chemotherapy for patients with unresectable bilobar colorectal liver metastases. Dig Surg 2008; 25: 387-393.

9. Nagino M, Kamiya J, Nishio H, et al. Two hundred forty consecutive portal vein embolizations before extended hepatectomy for biliary cancer: surgical outcome and long-term follow-up. Ann Surg 2006; 243: 364-372.

10. Abulkhir A, Limongelli P, Healey AJ, et al. Preoperative portal vein embolization for major liver resection: a meta-analysis. Ann Surg 2008; 247: 49-57.

11. Adam R, Laurent A, Azoulay D, et al. Two-stage hepatectomy: a planned strategy to treat irresectable liver tumors. Ann Surg 2000; 232: 777-785.

12. Lam VW, Laurence JM, Johnston E, et al. A systematic review of two stage hepatectomy in patients with initially unresectable colorectal liver metastases. HPB (Oxford) 2013; 15: 483-491.

13. Giuliante F, Ardito F, Ferrero A, et al. Tumor progression during preoperative chemotherapy predicts failure to complete 2-stage hepatectomy for colorectal liver metastases: results of an Italian multicenter analysis of 130 patients. J Am Coll Surg 2014; 219: 285-294.

14. Schadde E, Raptis DA, Schnitzbauer AA, et al. Prediction of mortality after ALPPS stage-1: an analysis of 320 patients from the International ALPPS Registry. Ann Surg 2015; 262: 780-785, discussion 785-786

15. Schadde E, Schnitzbauer AA, Tschuor C, et al. Systematic review and meta-analysis of feasibility, safety, and efficacy of a novel procedure: associating liver partition and portal vein ligation for staged hepatectomy. Ann Surg Oncol 2015; 22: 3109-3120.

16. Shindoh J, Vauthey JN, Zimmitti G, et al. Analysis of the efficacy of portal vein embolization for patients with extensive liver malignancy and very low future liver remnant volume, including a comparison with the associating liver partition with portal vein ligation for staged hepatectomy approach. J Am Coll Surg 2013; 217: 126-133, discussion 133-134.

17. Oldhafer KJ, Donati M, Jenner RM, et al. ALPPS for patients with colorectal liver metastases: effective liver hypertrophy, but early tumor recurrence. World J Surg 2014; 38: 1504-1509.

18. Choi YI, Moon HH, Shin DH. Two cases of ALPPS procedure: simultaneous ALPPS and colorectal resection and ALPPS pro- 
cedure for hepatic malignancy larger than 15 centimeter. Ann Hepatobiliary Pancreat Surg 2017; 21: 151-156.

19. Robles R, Parrilla P, López-Conesa A, et al. Tourniquet modification of the associating liver partition and portal ligation for staged hepatectomy procedure. Br J Surg 2014; 101: 1129-1134.

20. Chan AC, Pang R, Poon RT. Simplifying the ALPPS procedure by the anterior approach. Ann Surg 2014; 260: e3.

21. Donati M, Stavrou GA, Oldhafer KJ. Current position of ALPPS in the surgical landscape of CRLM treatment proposals. World J Gastroenterol 2013; 19: 6548-6554.

22. Hernandez-Alejandro R, Bertens KA, Pineda-Solis K, et al. Can we improve the morbidity and mortality associated with the associating liver partition with portal vein ligation for staged hepatectomy (ALPPS) procedure in the management of colorectal liver metastases? Surgery 2015; 157: 194-201.

23. Gauzolino R, Castagnet M, Blanleuil ML, et al. The ALPPS technique for bilateral colorectal metastases: three "variations on a theme". Updates Surg 2013; 65: 141-148.

24. de Santibañes M, Alvarez FA, Santos FR, et al. The associating liver partition and portal vein ligation for staged hepatectomy approach using only segments I and IV as future liver remnant. J Am Coll Surg 2014; 219: e5-9.

25. Schadde E, Malagó M, Hernandez-Alejandro R, et al. Monosegment ALPPS hepatectomy: extending resectability by rapid hypertrophy. Surgery 2015; 157: 676-689.

26. Steinbrück K, D’Oliveira M, Cano R, et al. Monosegmental ALPPS after bilateral hepatectomy. Ann Hepatol 2017; 16: 814817.

27. Maulat C, Suc B, Muscari F. Rescue ALPPS after portal embolization for colorectal metastases (with video). J Visc Surg 2018; 155: 77-78.

28. Björnsson B, Gasslander T, Sandström P. In situ split of the liver when portal venous embolization fails to induce hypertrophy: a report of two cases. Case Rep Surg 2013; 2013: 238675.

29. Vyas SJ, Davies N, Grant L, et al. Failure of portal venous embolization. ALPPS as salvage enabling successful resection of bilobar liver metastases. J Gastrointest Cancer 2014; 45: 233-236.

30. Tschuor C, Croome K, Sergeant G, et al. Salvage parenchymal liver transection for patients with insufficient volume increase after portal vein occlusion - an extension of the ALPPS approach. Eur J Surg Oncol 2013; 39: 1230-1235.

31. Machado MA, Makdissi FF, Surjan RC. Totally laparoscopic ALPPS is feasible and may be worthwhile. Ann Surg 2012; 256: e13.

32. Machado MA, Makdissi FF, Surjan RC, et al. Transition from open to laparoscopic ALPPS for patients with very small FLR: the initial experience. HPB (Oxford) 2017; 19: 59-66.

33. Conrad C, Shivathirthan N, Camerlo A, et al. Laparoscopic portal vein ligation with in situ liver split for failed portal vein embolization. Ann Surg 2012; 256: e14-15.

34. Vicente E, Quijano Y, Ielpo B, et al. First ALPPS procedure using a total robotic approach. Surg Oncol 2016; 25: 457.

35. Gringeri E, Boetto R, D’Amico FE, et al. Laparoscopic microwave ablation and portal vein ligation for staged hepatectomy (LAPS): a minimally invasive first-step approach. Ann Surg 2015; 261: e42-43.

36. Machado M, Surjan R, Basseres T, et al. Total laparoscopic reversal ALPPS. Ann Surg Oncol 2017; 24: 1048-1049.

37. Abdalla EK, Adam R, Bilchik AJ, et al. Improving resectability of hepatic colorectal metastases: expert consensus statement. Ann Surg Oncol 2006; 13: 1271-1280.

38. Oldhafer KJ, Stavrou GA, van Gulik TM. ALPPS - where do we stand, where do we go?: eight recommendations from the first international expert meeting. Ann Surg 2016; 263: 839-841.
39. Tanaka K, Endo I. ALPPS: short-term outcome and functional changes in the future liver remnant. Ann Surg 2015; 262: e88-89.

40. Ielpo B, Caruso R, Ferri V, et al. ALPPS procedure: our experience and state of the art. Hepatogastroenterology 2013; 60: 2069-2075.

41. Moris D, Ronnekleiv-Kelly S, Kostakis ID, et al. Operative results and oncologic outcomes of associating liver partition and portal vein ligation for staged hepatectomy (ALPPS) versus two-stage hepatectomy (TSH) in patients with unresectable colorectal liver metastases: a systematic review and meta-analysis. World J Surg 2018; 42: 806-815.

42. Gall TM, Sodergren MH, Frampton AE, et al. Radiofrequency-assisted liver partition with portal vein ligation (RALPP) for liver regeneration. Ann Surg 2015; 261: e45-46.

43. Oldhafer F, Ringe KI, Timrott K, et al. Monitoring of liver function in a 73-year old patient undergoing 'Associating Liver Partition and Portal vein ligation for Staged hepatectomy': case report applying the novel liver maximum function capacity test. Patient Saf Surg 2016; 10: 16.

44. Adam R, Imai K, Castro Benitez C, et al. Outcome after associating liver partition and portal vein ligation for staged hepatectomy and conventional two-stage hepatectomy for colorectal liver metastases. Br J Surg 2016; 103: 1521-1529.

45. Björnsson B, Sparrelid E, Røsok B, et al. Associating liver partition and portal vein ligation for staged hepatectomy in patients with colorectal liver metastases - intermediate oncological results. Eur J Surg Oncol 2016; 42: 531-537.

46. Lang H. ALPPS for colorectal liver metastases. J Gastrointest Surg 2017; 21: 190-192.

47. Schadde E, Ardiles V, Robles-Campos R, et al.; ALPPS Registry Group. Early survival and safety of ALPPS: first report of the International ALPPS Registry. Ann Surg 2014; 260: 829-36, discussion 836-838.

48. Brouquet A, Abdalla EK, Kopetz S, et al. High survival rate after two-stage resection of advanced colorectal liver metastases: response-based selection and complete resection define outcome. J Clin Oncol 2011; 29: 1083-1090.

49. Balzan S, Belghiti J, Farges O, et al. The "50-50 criteria" on postoperative day 5: an accurate predictor of liver failure and death after hepatectomy. Ann Surg 2005; 242: 824-828, discussion 828-829.

50. Aloia TA. Insights into ALPPS. Eur J Surg Oncol 2015; 41: 610611.

51. Cieslak KP, Olthof PB, van Lienden KP, et al. Assessment of liver function using $(99 \mathrm{~m})$ tc-mebrofenin hepatobiliary scintigraphy in ALPPS (associating liver partition and portal vein ligation for staged hepatectomy). Case Rep Gastroenterol 2015; 9: 353-360.

52. Truant S, Baillet C, Deshorgue AC, et al. Drop of total liver function in the interstages of the new associating liver partition and portal vein ligation for staged hepatectomy technique: analysis of the "auxiliary liver" by HIDA Scintigraphy. Ann Surg 2016; 263: e33-e34.

53. Sparrelid E, Jonas E, Tzortzakakis A, et al. Dynamic evaluation of liver volume and function in associating liver partition and portal vein ligation for staged hepatectomy. J Gastrointest Surg 2017; 21: 967-974.

54. Rahbari NN, Garden OJ, Padbury R, et al. Posthepatectomy liver failure: a definition and grading by the International Study Group of Liver Surgery (ISGLS). Surgery 2011; 149: 713-724.

55. Linecker M, Stavrou GA, Oldhafer KJ, et al. The ALPPS risk score: avoiding futile use of ALPPS. Ann Surg 2016; 264: 763-771.

56. Alghamdi T, Viebahn C, Justinger C, Lorf T. Arterial blood supply of liver segment IV and its possible surgical consequences. Am J Transplant 2017; 17: 1064-1070. 
57. Li J, Girotti P, Königsrainer I, et al. ALPPS in right trisectionectomy: a safe procedure to avoid postoperative liver failure? J Gastrointest Surg 2013; 17: 956-961.

58. Vivarelli M, Aldrighetti LA, Robles Campos R, et al. High mortality after ALPPS for perihilar cholangiocarcinoma: case-control analysis including the first series from the international ALPPS registry. HPB (Oxford) 2017; 19: 381-387.

59. Shoup M, Gonen M, D’Angelica M, et al. Volumetric analysis predicts hepatic dysfunction in patients undergoing major liver resection. J Gastrointest Surg 2003; 7: 325-330.

60. Ferrero A, Viganò L, Polastri R, et al. Postoperative liver dysfunction and future remnant liver: where is the limit? Results of a prospective study. World J Surg 2007; 31: 1643-1651.

61. Guglielmi A, Ruzzenente A, Conci S, et al. How much remnant is enough in liver resection? Dig Surg 2012; 29: 6-17.

62. Croome KP, Hernandez-Alejandro R, Parker M, et al. Is the liver kinetic growth rate in ALPPS unprecedented when compared with PVE and living donor liver transplant? A multicentre analysis. HPB (Oxford) 2015; 17: 477-484.

63. Ratti F, Schadde E, Masetti M, et al. Strategies to Increase the resectability of patients with colorectal liver metastases: a multi-center case-match analysis of ALPPS and conventional two-stage hepatectomy. Ann Surg Oncol 2015; 22: 1933-1942.

64. Matsuo K, Murakami T, Kawaguchi D, et al. Histologic features after surgery associating liver partition and portal vein ligation for staged hepatectomy versus those after hepatectomy with portal vein embolization. Surgery 2016; 159: 1289-1298.

65. Chia DKA, Yeo Z, Loh SEK, et al. Greater hypertrophy can be achieved with associating liver partition with portal vein ligation for staged hepatectomy compared to conventional staged hepatectomy, but with a higher price to pay? Am J Surg 2018; 215: 131-137.

66. Røsok BI, Björnsson B, Sparrelid E, et al. Scandinavian multicenter study on the safety and feasibility of the associating liver partition and portal vein ligation for staged hepatectomy procedure. Surgery 2016; 159: 1279-1286.

67. Schnitzbauer AA, Schadde E, Linecker $M$, et al. Indicating ALPPS for colorectal liver metastases: a critical analysis of patients in the International ALPPS registry. Surgery 2018; 164: 387-394.

68. Yamanaka N, Okamoto E, Kawamura E, et al. Dynamics of normal and injured human liver regeneration after hepatectomy as assessed on the basis of computed tomography and liver function. Hepatology 1993; 18: 79-85.

69. Nagasue N, Yukaya H, Ogawa Y, et al. Human liver regeneration after major hepatic resection. A study of normal liver and livers with chronic hepatitis and cirrhosis. Ann Surg 1987; 206: 30-39.

70. Vivarelli M, Vincenzi P, Montalti R, et al. ALPPS procedure for extended liver resections: a single centre experience and a systematic review. PLoS One 2015; 10: e0144019.

71. Lisotti A, Azzaroli F, Buonfiglioli F, et al. Indocyanine green retention test as a noninvasive marker of portal hypertension and esophageal varices in compensated liver cirrhosis. Hepatology 2014; 59: 643-650.

72. Lisotti A, Azzaroli F, Cucchetti A, et al. Relationship between indocyanine green retention test, decompensation and survival in patients with Child-Pugh A cirrhosis and portal hypertension. Liver Int 2016; 36: 1313-1132.

73. Fan ST, Lai EC, Lo CM, et al. Hospital mortality of major hepatectomy for hepatocellular carcinoma associated with cirrhosis. Arch Surg 1995; 130: 198-203.

74. Narita M, Oussoultzoglou E, Ikai I, et al. Right portal vein ligation combined with in-situ splitting induces rapid left lateral liver lobe hypertrophy enabling 2-staged extended right hepatic resection in small-for-size settings. Ann Surg 2012; 256: e7-8; author reply e16-17.

75. Donati M, Basile F, Oldhafer KJ. Present status and future perspectives of ALPPS (associating liver partition and portal vein ligation for staged hepatectomy). Future Oncol 2015; 11: 22552258.

76. Buac S, Schadde E, Schnitzbauer AA, et al. The many faces of ALPPS:surgical indications and techniques among surgeons collaborating in the international registry. HPB (Oxford) 2016; 18: $442-448$

77. Lai EC, Fan ST, Lo CM, et al. Anterior approach for difficult major right hepatectomy. World J Surg 1996; 20: 314-317, discussion 318

78. Chan AC, Poon RT, Lo CM. Modified anterior approach for the ALPPS procedure: how we do it. World J Surg 2015; 39: 28312835.

79. Ardiles V, Schadde E, Santibanes E, Clavien PA. Commentary on "Happy marriage or "dangerous liaison": ALPPS and the anterior approach". Ann Surg 2014; 260: e4.

80. Schlegel A, Lesurtel M, Melloul E, et al. ALPPS: from human to mice highlighting accelerated and novel mechanisms of liver regeneration. Ann Surg 2014; 260: 839-846, discussion 846-847.

81. Petrowsky H, Györi G, de Oliveira M, et al. Is partial-ALPPS safer than ALPPS? A single-center experience. Ann Surg 2015; 261: e90-e92.

82. Cai YL, Song PP, Tang W, Cheng NS. An updated systematic review of the evolution of ALPPS and evaluation of its advantages and disadvantages in accordance with current evidence. Medicine (Baltimore) 2016; 95: e3941.

83. Chan ACY, Chok K, Dai JWC, Lo CM. Impact of split completeness on future liver remnant hypertrophy in associating liver partition and portal vein ligation for staged hepatectomy (ALPPS) in hepatocellular carcinoma: complete-ALPPS versus partial-ALPPS. Surgery 2017; 161: 357-364.

84. Sandström P, Røsok BI, Sparrelid E, et al. ALPPS improves resectability compared with conventional two stage hepatectomy in patients with advanced colorectal liver metastasis: results from a Scandinavian multicenter randomized controlled trial (LIGRO trial). Ann Surg 2018; 267: 833-840. 